\title{
Discerning the Intimacies of Intertextuality: A.K. Ramanujan's Hyphenated Cosmopolitan Approach to Translation Theory and Practice
}

\author{
A. S. Dasan
}

\begin{abstract}
A.K. Ramanujan (1929-1993) -- poet, philologist, folklorist, translator, and playwright, wrote widely in a number of genres spanning across disciplines. Well versed in five languages -- English, Kannada, Tamil, Telugu, and Sanskrit, his theoretical and aesthetic assimilations and articulations, vis-à-vis translation, argued for non-standardized dialectics, context-sensitive and pragmatic hermeneutics and sensibilities and glocalized aesthetics espousing at the same time a cosmopolitan approach to translation theory and practice. This paper, originally delivered as the Keynote Address in a UGC-sponsored National Seminar on "A.K. Ramanujan and the Postcolonial Theory and Practice of Translation" at KLE Society's Lingaraj College (Autonomous), Belgaum, Karnataka, in March, 2014, is a revised version wherein Ramanujan's approach to translation is viewed as a bridge-making endeavour between abstractions and experience so as to discern the affinities between aham (the interior) and puram (the exterior) vis-à-vis intertextualities.*
\end{abstract}

A.K. Ramanujan (AKR) was a master-story teller whose writing-career spanned more than four decades. His literary life may be summed up as 'a story in search of an audience' across disciplines to use his own phrase.' The sagacity with which he tells every story, be it through his poems or translated texts of folktales, not only delights us, readers across disciplines, but also

*

A.S.Dasan, PhD, formerly Chairman, Dept. of English, and FounderDirector of the Centre for Proficiency Development and Placement Services (CPDPS), University of Mysore, is currently Professor of English and Director, Tap-Me Academy: Shukrodaya's School of Proficiency for HRD, Mysore. Correspondence to: asdmysore@gmail.com 
inspires us to look at him as 'an akshayapatra', with the fullness of the pot never getting emptied, to quote Girish Karnad ${ }^{2}$ whose close contacts with AKR enabled him to compliment that way at the end of his 'A.K. Ramanjuan Memorial Lecture', the first one organised at Ramjas College, University of Delhi on $21^{\text {st }}$ March, 2012.

Though Mathematics, which was his father's and his brother's profession, was not his cup of tea, AKR's story-telling ways, his context-sensitive translating and narrative techniques and his audience-centred sensitivity manifest an algebraic precision. One Kannada text that spontaneously comes to my mind is:

\section{Ullavaru,}

Shivalaya Va Maaduvaru:

The rich, will make temples for Siva.

Naanuenu Maadali,

Badavanaiya:

What shall I,

a poor man, do? ${ }^{3}$

Born in 1929 in a Hindu Brahmin-family with Tamil as mother tongue, schooled in Kannada, graduated in English, married to a Malayalee Christian, and professionally focused on a diachronic and cosmopolitan outlook and worldview leaning towards Buddhism, AKR's philological versatility and academic research ranged across five languages -- Tamil, Kannada, Telugu, Sanskrit, and English -- and took him beyond the frontiers of India, his motherland, motivating him to ignite and harness his intellectual energies through the green pastures of America, the land of critical literacy and promising future for the meritorious. For more than forty years, AKR pursued resolutely and vibrantly multifaceted careers - as a bilingual poet, pragmatic-formalistlinguist, practising-translator, and insightful folklorist, involved 
ethnographer, intermittent novella-writer, and playwright, pursuing all these careers simultaneously, almost like his fulltime preoccupations. He wrote books on both classical and modern literatures and convincingly argued for non-standardized dialectics, context-sensitive and pragmatic hermeneutics and sensibilities and glocalized aesthetics. From this point of view, his trend-setting contribution towards cosmopolitan approach to translation theory and practice is of immense value. Out of all the careers he pursued till his death in 1993, writing as a bilingual poet was his foremost vocation. But the one that bridged his literary and scholarly careers was his vocation as translator which is the prime focus of this paper.

Before I delve deep into the prime focus, I wish to clarify that bracketing AKR as a translator within the 'postcolonial turn' as if he were a postcolonial ideologue would be rather a narrow reading, if not a misreading, as AKR's theory and practice of translation does not fit well within that bracket. AKR neither subscribed to nor restricted himself to the 'postcolonial turn', the turn either as an ideological movement, meaning political act that includes resistance and transformation against colonial/ hegemonic schema, or as a temporal marker, meaning periodicity. He was neither a postcolonial ideologue in the sense as espoused by Henry Louis Gates, Jr., Gayatri C. Spivak and Homi K. Bhabha nor he belonged to the Stephanian group of diasporic or postcolonial writers. His story-telling or theory and practice of translation did not focus on examining the relationships between language and power across cultural boundaries, or redefining the meanings of cultural or ethnic identity. Ideology or postcolonial discoursing was not his forte.

In her book, Siting Translation (1992), Tejaswini Niranjana slams AKR, with reference to his translated text, Speaking of Shiva (1973), for his 'reliance on formalist and modernist frameworks', critiquing that such frameworks 'are inappropriate to the task of translating poetry'. Unfortunately, Tejaswini Niranjana critiques AKR from an ideological bracket alone. It is not fair. The way Vinay 
Dharwadker has tackled Niranjana's criticism is worth reading. ${ }^{4}$ It is sufficient and relevant here to note that the periodicity of AKR's vocation as a translator begins soon after his entry into the University of Chicago (1962) after having passed through the University of Indiana for his doctoral studies, and much before the postcolonial theoretical ideologues emerged in the postcolonial scenario with The Empire Writes Back (1989) and the much talked about 'cultural turn' in translation studies (Susan Bassnett and André Lefevere, 1990). The context-sensitivity AKR is conscious of in his translation endeavours and exercises is much more pragmatic, synthesizing, synergizing and cosmopolitan than merely being viewed as part of postcolonial-turns or contextualextremes. Today, the term, 'postcolonial', itself, is a withering concept, and therefore, when we read AKR, we need to move beyond postcolonial angularities and limitations.

To come back to the focus of this paper, AKR's approach to translation was a conscious and perennial quest for bridgemaking between abstractions and experience towards discerning the affinities between aham (the interior) and puram (the exterior) vis-à-vis intertextualities. The selection of 'Thevakulathär's love-lyric from Kurunthokai of Tamil Sangam Poetry), which is translated and placed as the opening lines in his book, The Interior Landscape, cited later in another context in this paper, may be considered as the touch-stone and hallmark of his approaches to translation. The number of Introductions and Commentaries AKR wrote to his own collections of poems and other translated texts serve, as Karnad notes, also as windows allowing fresh-air towards understanding and appreciating his pragmatic approach to translation theory and practice. They connect readers with his perennial quest for discerning the intimacies of intertextuality and 'aesthetic heterodoxy' in terms of linguistic, semiotic and semantic categories, the interior (aham) and the exterior (puram) and the visual and the aural forms which, as Vinay Dharwadker comments, cumulatively 'bring together an unparalleled variety of languages, texts, genres, literatures, historical periods, and past and present cultures.'. Vinay Dharwadker adds that AKR 
'translated literary works mainly from Kannada and Tamil into English, but also, less extensively, from English into Kannada and, with the help of collaborators, from Malayalam, Telugu, Marathi, and Sanskrit into English'. 'He focused his attention on verse as well as prose, rendering epic and classical poetry from the ancient period (chiefly works composed between about 500 B.C. and 500 A.D.), early and late poetic texts from the middle period -- from the eighth to the eighteenth centuries, and poems, short stories, novelistic fiction, and numerous folktales from the modern period -- the nineteenth and twentieth centuries'. The gentle but radical departures he made in the course of rendering translations not only dismantled existing standardized principles but also delighted readers across disciplines, cultures and continents by virtue of their creative and plurisignifying possibilities.

AKR's classroom and formal lectures, his scholarly articles, his conference papers, and translation exercises ranged with 'effortless expertise over linguistics, anthropology, history of religions, folklore, and literary studies, usually covering several South Asian, British, American, and European discursive traditions reveal his predilection for foregrounding intertextuality and celebrating cosmopolitanism. His essays, "The Indian Oedipus" (1983), "Telling Tales" (1989), "Where Mirrors Are Windows" (1989), "Toward a Counter-System: Women's Tales" (1991), and "Three Hundred Rãmãyanas" (1991) may be cited here as instances wherein his interdisciplinary, critical, and interpretive engagements are evident.

The cosmopolitanism he was committed to as a person, author, scholar, teacher, and translator had something to do with his innate tastes, rasas and gunas. Though brought up in the ambience of Mysore Brahminical Puritanism, the places he traversed and located both in India and abroad vis-à-vis his professional engagements and commitments served as matrices for practising cosmopolitan aesthetics. His balanced contextsensitivity evident in his translations which include Interior Landscapes: Love Poems from a Classical Tamil Anthology (1967), 
Speaking of Siva (1973), Hymns for the Drowning (1981), and A Flowering Tree and Other Oral Tales from India (1997) foregrounds intertextual influences coming from varied sources, especially non-Sanskrit Indian regional literatures (Tamil Sangam Poetry, for instance) and establishes the complex sources and resources of creativity and poetry. It is within these intertextual matrices that 'the sublime and the earthy, the outstanding and the ordinary, the poetic and the prosaic, the tentative and the definitive interface in a catalysing fertility' exuding the contours and the fragrance of his open and inclusive secular imagination, ushering in certain modernist secularist ethos. This was his way of celebrating cosmopolitanism that connects the linguistic formalism with the semantics which connect the aham and the puram as enunciated in Sangam Poetry and illustrated in his translations such as the one quoted here below:

தலைமகன் சிறைப்புறமாக, அவன் வரைந்து கொள்வது வேண்டி, தோழி இயற் பழித்தவழி, தலைமகள் இயற்பட மொழிந்த து:

What the heroine said about her love for the hero, at the intervention of her friend, so that the hero, who was listening nearby, could hear:

நிலத்தினும் பெரிதே; வானினும் உயர்ந்தன்று;

Nilathinum peridhe; vaninum uyardhanru;

நீரினும் ஆர் அளவின்றே - சாரல்

Neerinum aar alavindre-saaral

கருங் கோல் குறிஞ்சிப்பூக் கொண்டு

karunkòl kuriñci puukondu

பெருந்தேன் இழைக்கும் நாடனொடு நட்பே perundhaen laikkum naadanodu natpae.

--- ---தேவகுலத்தார்: குறுந்தொகை 3, குறிஞ்சித் திணை 
Bigger than earth, certainly, higher than the sky, more unfathomable than the waters is this love for this man

\author{
of the mountain slopes \\ where bees make rich honey \\ from the flowers of the kurinji \\ that has such black stalks.
}

- 'Thevakulathaar's Kuriñci-Thogai, $3^{6}$

This medieval and complex Tamil text consists of a single long sentence allowing considerable freedom in the ordering of the parts of the sentence wherein the theme of love, a human feeling, is connoted and placed only at the end of the sentence. Sangam poets were deft at capturing the spirit of the poetic moment within a language of brevity. Brevity was the lifeline of their poetry in terms of both semiotics and semantics. In fact, the Tamil text cited above integrates the exterior analogy (bees gathering honey from kurinji flowers at the mountain slopes and taking it to the mountain top) with the logic of the interior thought (the union of two hearts coming from two different places and merging into one) in such a fine rhetoric brevity that it is a shining illustration of intertextuality that paves the way for hermeneutics with multiple connotations. The intertextuality AKR was conscious of foregrounds notions of relationality, interconnectedness and interdependence in a literary text. It implies 'a paradoxical nature of the discursive space that makes a text intelligible', to rephrase the language Julia Kristeva uses when she defines intertextuality. Within this intelligibility, diachrony is transformed into synchrony. This is what happens in the Tamil text quoted above and in the translation by AKR. The intertextualities ingrained in the text cumulatively exude the fragrance of literary excellences vis-à-vis 'the power of imagination' (கற்பனை), 'the language of rhetoric' 
(சொல்லாட்சி), 'the use of analogy (உவமை), 'the logic of inner thought' (உள்ளுறை), and 'the technique of indirection or suggestion' (இறைச்சி). The task of rendering of the theme of love, the virgin's love 'moving towards the black-stalked kuriñci, acting out by analogue the virgin's progress from abstraction to experience,' into idiomatic English with such terseness and compactness as it is in Tamil is a tough one indeed. The translation done by AKR in terms of conceptualization, style, and presentation similar to that in the original text is a superb one for the way it ensures that the structure of the language (the exterior) 'moves from the earth, sky and water through the slopes, bees and flowers of the mountain country, all converging just to convey the deeper truth, the inner thought (the interior), namely the lady's love as 'bigger than the earth and higher than the sky'. That is the quintessence of the inner and figural landscape of the Tamil poetic text, and AKR conveys it succinctly.

This complex and terse translation is an instance which indicates why and how the translator has a responsibility to respond visually to the aural form of the original in terms of aham (the inner core) and puram (the outer core). Here, the whole universe permeates the visual form spiralling down to the details of the kurinji flower (Strobilanthes Kunthiana). The way AKR connects the exterior and the interior, the particular and the universal, the mundane and the contemplative, and the local and the cosmic shows the distinctive style of his translation-practice. This is the Indic touch AKR is particular to ingrain within the target language, and the source language text he has chosen yields to such rendering. His axiom, 'only a poet can translate a poem' can be extended to include, 'only a poet with such Indic sensitivity and sensibility can translate a poem' this way. This justifies the title of this paper vis-à-vis intimacies of intertextuality and hyphenated cosmopolitanism.

The Indic touch of eclectic accommodativeness and qualified universalism (Indian ways of celebrating differences and diversities) is the hallmark of his writing-career. It was his 
conscious choice and commitment in the course his translational activities too. His stupendous contributions to Indian subcontinent studies and South Asian studies serve as mirrors and windows for a multidimensional anthology of reflections on crossdisciplinary perspectives, complexities of culture, and subtleties of Indic thought. His translations of select classical and bhakti poetry in Tamil, Veerasaiva Vachanas in Kannada, bhakti literature in Telugu and oral narratives strike a fine balance between source and target languages and between the author's interest and his own interest. A reading of Speaking of Shiva and two of his select essays, "Is there an Indian way of Thinking?" (1990) and "Three Hundred Ramanayanas" (1991) would vouchsafe and confirm such a perception.

True, as Vinay Dharwadker notes, AKR may have 'developed his ideas of outer and inner poetic forms from two different sources' - Noam Chomsky's concept of deep and surface structures and Roman Jakobson's distinction between 'verse instance' and 'verse design'. Looking for similarities between AKR's approach and Julia Kristeva's distinction between 'phenotext' (the manifest text) and 'genotext' (the innate signifying structure) may be tenable too. But, what is more true is that his fluency in English and in the disciplines of linguistics and anthropology gave him his outer form while personal and career-focused predilections for Tamil, Kannada and other Indian folklore forms gave him his inner self. He was particular that 'these two forms had to be in dialogue with each other'. The dialogic consort and connection he ingrains in the course of translating a text, to quote Vinay Dharwadker again, "evolves into an open-ended, multi-track process, in which translator, author, poem and reader move back and forth between two different sets of languages, cultures, historical situations and traditions". 8 The multiple levels his theory and practice of translation emphasize consort and interact, just like the process involved in a bricolage, without collapsing onto each other, in such a way that they ensure that 'sentences have priority over words, and discursive structures have priority over sentences'. In other words, in his view, words have relevance within sentences and 
sentences within discursive structures. The multi levels converge towards enabling readers to enjoy and experience a holistic and synergic reading of the translated text.

AKR's selections of literary texts - poems and stories - for the purpose of translation, done during the four decades of his active involvement in translation-activities, stand as a collective testimony to this dialogic and context-sensitive connections. They ensure that translation is a conscious heteroglassic exercise that can 'energize' readers to discern the scintillating polyphony a great translated text ushers in without missing or bypassing the inner logic of the original text. Such an exercise widens not only translator's 'translating consciousness' vis-à-vis 'inter-lingual language systems' and 'inter-lingual synonymy' to use the phrases of G.N.Devy ${ }^{9}$, but also readers' mirroring of the inner self to the world.

With regard to AKR's position on the question of equivalence, AKR states, with reference to Kampan's retelling of Valmiki's Rãmãmyana, that 'iconic fidelity to the original may be a great value in the West' but we, in India / Asia, 'rejoice in the similarity and cherish and savour the differences'. His essay, "Three Hundred Rãmãmyanas", is a fine example of such cultural and aesthetic heterodoxy. Practising and prospective translators may be reminded that AKR was not an imitator or follower of the Western metaphysics of translation -- readers may remember Hillis Miller's statement, namely 'translation is a wandering existence in a perpetual exile', a post-Babel crisis -- which has an obsession with the quest for equivalence, a quest linked to the Christian theological concern vis-à-vis paradise lost and paradise to be regained. To him, what matters ultimately is the rasa-anubhava, Bharata's shaping principle that gives the work of art, original or translated text, its distinctive quality, its authentic art-emotion.

In the light of these observations, let me touch on the basic principles of AKR's theory and practice of translation: 


\section{Author-Translator-Reader Relationship to be Maintained:}

It is good to keep in mind that AKR is not a poststructuralisttranslator to do away with the intimacies of the author-translatorreader relationship. He does not indulge in an ad-infinitum exercise 'exploding the binary opposition between the original and the target language'. He does not project himself as an elitist 'exaggerating the indeterminacy in meaning-making'. He does not advocate an autonomous or agentless textuality or intertextuality that tends to ignore or reject humanistic aesthetics. He is aware of the fact that conflict-ridden situations would arise but it is the responsibility of the translator to reconcile through contextsensitivity without yielding to extremes. There should be a balance between transmission of the original and expression of the translator in resonance with the target context and text striking a rhythmic inner landscaping balance between a source-oriented translation and target-oriented without privileging one against the other. This resonance should pave the way for rasa-anubhaval ananda in the ambience of the intimacies of intertextuality. Emotive proximities and affinities within the triangle of authortranslator-reader relationship could help discern the intimacies of intertextuality better.

\section{Translation involves a reader-sensitive cross-cultural transmission:}

The intertextual network implied in the task of translation opens out a multi-track process in terms of cross-cultural transmission and expression. The author's source text needs to be transmitted in the context of the contemporary reader. While taking a particular text from one culture into another, the translator also translates the reader from the second culture into the first one keeping in mind the expectations of the reader. The reader expects reliability of representation and delight and aesthetic pleasure. This is what AKR does in his translation (1976) of U.R. Ananthamurthy's Samskara (1965). In other words, as Vinay Dharwadker adds, the process of translation, according to AKR, 
'energizes' everyone concerned. It should 'bridge the divide of cultures, languages and years between the text and the reader'.

\section{Socio-linguistic Approach:}

Critiquing and moving beyond the monolithic Sanskritgrammatology and Tamil-brahminical dialects, AKR tries to legitimize a vast variety of non-Sanskrit / non-monolithic linguistic dialects of India which suits his linguistic innovation in the course of translating a text. This is his way of responding to the expectations of various social groups among his readers in India and Indian diasporic readers outside India. This thrust is abundantly clear in his essay co-authored with W. Bright, "Sociolinguistic Variation and Language Change" (1964). This is why AKR recommends 'phrase-to phrase' rather than 'word to word' translation without losing sight of the inner logic of the original and suggests that parallelism rather complete equivalence is of significant value in terms of relationality between the source text and the target text. This implies that multiple translations of one and the same text are possible, all the more because of the complexity involved when one takes into account the diachronic diversity of the subcontinent's cultures.

\section{Pragmatic and Inclusive Aesthetic Approach:}

As a practitioner, AKR was more focused on 'the back of the embroidery' than on the finished product which becomes the object of the reader's delight. Parts contribute to the whole. Parts are as fascinating as the whole. This explains his sense of pragmatism. His linguistic-formalist approach cannot be separated from his pragmatic approach to art, literature and culture. He insists more on the principles of construction (the process) than on the product.

\section{Hyphenated Cosmopolitanism:}

In AKR's paradoxical worldview, no language is pure and 
yet, every language is potentially grammatical and grammatically a complete system. In the process of translation, what matters is the network of relations. The 'inner logics' ingrained within the two languages / cultures of the texts in question ensure that irrationalities of external boundaries are overcome by 'the leap of imagination' the translator is capable of. From this point of view, AKR felt home everywhere. ${ }^{10}$ He did not have problems like the postcolonial diasporic writers who write with their angst on 'roosts', 'exile' and 'memory' or with a predilection for resistance. Yet, the Indianness to which he was personally, professionally and aesthetically committed never deserted him in his quest for constructive cosmopolitanism. His constructive cosmopolitanism was an irresistible and ceaseless poetry of connections which could tie relations between and across nations, cultures and languages, where 'nothing would be lost', celebrating diachronic rather than synchronic multiculturalism. His poetry of connections is not 'predicated by any one monolithic, Eurocentric, unipolar direction'. This sums up his 'relativist multicultural approach' that hyphenated his ideas of cosmopolitanism and restricted universalism in the backdrop of the complexities and intimacies of intertextuality associated with the art and task of translation.

To conclude, I have embraced more or less the known paths than the new ones in this paper. It is only a modest proposal to highlight the Indic affinities of AKR vis-à-vis his theory and practice of translation for further provocations and explorations. There are quite a few more roads not yet taken. The times we live in are prone to binary oppositions and conflicts, 'circling the square or squaring the circle.' AKR's approaches to translation counter such vanities. Mere binary approaches in the name of poststructuralism or postcolonialism may not take us anywhere beyond perpetuating conflict-ridden contexts and situations. We need to think like an ecologist who sees the value of boundaryless roots and routes consorting together beneath the soil and yet retaining their own uniqueness, fragrance and fruit-bearing abilities to the delight of onlookers and consumers. Let the spirit of ecology strike at the penury of translators who may struggle to 
possess the sense of discernment to see the power and beauty of the presence of balanced context-sensitive intuition, 'the third language', guiding them. The intuitive 'third language', I mean here, is the universal language, audible to ecologists, seers, and poets who think and see like seers, that transcends differences and barriers and builds bridges of understanding between the source language and the target language in the ambience of authortranslator-reader relationship kept live by context-sensitivities. Such a notion requires another keynote address, or a paper to be written.

\section{REFERENCES}

1. Ramanujan, A. K. Ed. Folktales from India, Oral Tales from Twenty Indian Languages. Penguin Books 1991. Readers may be familiar with AKR's telling of a Telugu folktale, called "A Story in Search of an Audience" (narrated by K.Katayani in Hyderabad in 1988, and orally translated by V. Narayana Rao).

2. Karnad, Girish.

"A.K. Ramanujan Memorial Lecture." Ramjas College, University of Delhi. 21st March, 2012. South Asia Citizens Web: http://www.sacw. net/article4762.html

3. Ramanujan, A. K.

Speaking of Siva. Penguin, 1973. Vachana Sahitya: 'The Temple and the Body' by Basava (11341196) -Trans. by AKR.

4. Dharwadker, Vinay. “A. K. Ramanujan's Theory and Practice of Translation" in 
Post-colonial Translation: Theory and Practice, Ed. By Bassnett and Trivedi, 1999. Pp. 114-140

5. Ibid.

6. Ramanujan, A. K.

Ed. and Transl. The Interior Landscape - Classical Tamil Love Poems. New York Review of tBooks. 1967.

7. Krishna, Nakul. "The Heron and the Lamprey." http://thepointmag.com/2014/criticism/theheron-and-the-lamprey

8. Dharwadker, Vinay.

"A. K. Ramanujan's Theory and Practice of Translation."

9. Devy, G.N. In Another Tongue - Essays on Indian English Literature. Lang, Peter Publishing. 1993.

10. Dharwadker, Vinay.

$$
\begin{aligned}
& \text { "Postcolonial Cosmopolitanism: A Note } \\
& \text { on A.K. Ramanujan's Theory and Practice } \\
& \text { of Criticism and Translation." } 1994 .
\end{aligned}
$$

